\title{
Effectiveness of self-management support interventions for people with comorbid diabetes and chronic kidney disease: a systematic review and meta-analysis
}

Edward Zimbudzi ${ }^{1,2}$ D, Clement Lo ${ }^{1,3}$, Marie L. Misso ${ }^{1}$, Sanjeeva Ranasinha ${ }^{1}$, Peter G. Kerr ${ }^{2,4}$, Helena J. Teede ${ }^{1,3}$ and Sophia Zoungas ${ }^{1,3,5^{*}}$

\begin{abstract}
Background: Self-management support interventions may potentially delay kidney function decline and associated complications in patients with comorbid diabetes and chronic kidney disease. However, the effectiveness of these interventions remains unclear. We investigated the effectiveness of current self-management support interventions and their specific components and elements in improving patient outcomes.

Methods: Electronic databases were systematically searched from January 1, 1994, to December 19, 2017. Eligible studies were randomized controlled trials on self-management support interventions for adults with comorbid diabetes and chronic kidney disease. Primary outcomes were systolic blood pressure, diastolic blood pressure, estimated glomerular filtration rate, and glycated hemoglobin. Secondary outcomes included self-management activity, health service utilization, health-related quality of life, medication adherence, and death.

Results: Of the 48 trials identified, eight studies (835 patients) were eligible. There was moderate-quality evidence that self-management support interventions improved self-management activity (standard mean difference $0.56,95 \% \mathrm{Cl} 0.15$ to $0.97, p<0.007)$ compared to usual care. There was low-quality evidence that self-management support interventions reduced systolic blood pressure (mean difference $-4.26 \mathrm{mmHg}, 95 \% \mathrm{Cl}-7.81$ to $-0.70, p=0.02$ ) and glycated hemoglobin (mean difference $-0.5 \%, 95 \% \mathrm{Cl}-0.8$ to $-0.1, p=0.01$ ) compared to usual care.

Conclusions: Self-management support interventions may improve self-care activities, systolic blood pressure, and glycated hemoglobin in patients with comorbid diabetes and chronic kidney disease. It was not possible to determine which self-management components and elements were more effective, but interventions that utilized provider reminders, patient education, and goal setting were associated with improved outcomes. More evidence from high-quality studies is required to support future self-management programs.
\end{abstract}

Systematic review registration: PROSPERO CRD42015017316.

Keywords: Chronic kidney disease, Diabetes, Interventions, Self-management, Systematic review, Meta-analyses

\footnotetext{
* Correspondence: sophia.zoungas@monash.edu

${ }^{1}$ Monash Centre for Health Research and Implementation, School of Public Health and Preventive Medicine, Monash University, 43-51 Kanooka Grove, Clayton, Melbourne, Victoria, Australia

${ }^{3}$ Diabetes and Vascular Medicine Unit, Monash Health, Melbourne, Victoria,

Australia

Full list of author information is available at the end of the article
}

(C) The Author(s). 2018 Open Access This article is distributed under the terms of the Creative Commons Attribution 4.0 International License (http://creativecommons.org/licenses/by/4.0/), which permits unrestricted use, distribution, and reproduction in any medium, provided you give appropriate credit to the original author(s) and the source, provide a link to the Creative Commons license, and indicate if changes were made. The Creative Commons Public Domain Dedication waiver (http://creativecommons.org/publicdomain/zero/1.0/) applies to the data made available in this article, unless otherwise stated. 


\section{Background}

The prevalence of diabetes is on the rise globally, driven primarily by the increasing incidence of type 2 diabetes in the setting of increasing overweight and obesity [1]. The International Diabetes Federation estimated that 415 million adults (aged 20-79 years) had diabetes in 2015 and 5 million deaths were attributable to diabetes and the total global health expenditure due to diabetes was 673 billion US dollars [2]. By 2040, the number of adults with diabetes (aged 20-79 years) is expected to rise to 642 million [2]. The dramatic increase in diabetes is associated with a myriad of diabetes-related complications such as cardiovascular disease, renal failure, blindness, and lower limb amputation [3].

Chronic kidney disease (CKD) is one of the commonest diabetes-related complications. Worldwide, current estimates suggests that over 500 million people have CKD, with the majority (80\%) of those people living in low- and middle-income countries [4] and diabetes contributes to $30-40 \%$ of all cases of end-stage renal disease (ESRD) [5]. In developed countries, diabetes accounts for $50 \%$ of cases of treated ESRD [6]. As the prevalence of diabetes increases, the incidence of CKD is expected to increase.

Co-morbid diabetes and CKD is associated with an increased risk of a range of adverse outcomes including increased mortality [7], low health-related quality of life [8], and increased health service utilization [9]. Self-management support interventions have generated considerable interest in the management of CKD as a means of helping to improve risk factors and slow disease progression [10]. However, the effects of self-management strategies for those with co-morbid diabetes and CKD are largely unknown [11]. Many current approaches to self-management for patients with both diabetes and CKD are based on interventions for single conditions rather than for patients with complex multimorbidity [11]. Additionally, there is a huge diversity of potential self-management support interventions which have been trialed making it difficult for health care providers to select the most pragmatic and effective interventions. To date, there has been no systematic review of the literature examining the effectiveness of self-management support interventions in people with both diabetes (type 1 or type 2) and CKD.

To address this, we undertook a systematic review, which sought to answer the following questions:

1. How effective are self-management support interventions in improving patient-reported and clinical outcomes in adults with comorbid diabetes and CKD?

2. Which specific self-management components and elements are associated with improved outcomes for patients with comorbid diabetes and CKD?

\section{Methods}

The conduct of this review was guided by the Cochrane Handbook for Systematic Reviews of Interventions [12] and conforms to the reporting guidelines of the Preferred Reporting Items for Systematic Reviews and Meta-Analyses (PRISMA) statement recommendations [13]. The protocol of this systematic review was registered on PROSPERO 2015 (registration number CRD42015017316) [14] and published [15].

\section{Selection criteria}

Table 1 presents the Population, Intervention, Comparison, and Outcome (PICO) framework established a priori to include and exclude studies for this systematic review.

\section{Participants}

This review considered studies of people with both diabetes (type 1 or type 2) and CKD. CKD was defined as a sustained decrease in estimated glomerular filtration rate (eGFR) to levels less than $60 \mathrm{~mL} / \mathrm{min} / 1.73 \mathrm{~m}^{2}$ for a period of 3 months or longer [16]. In studies where the inclusion criteria were not clear, we sought clarification from the corresponding authors and such studies were excluded if we could not get verification.

\section{Interventions}

For the purpose of this review, self-management support was defined as "the systematic provision of education and supportive interventions by health care staff to increase patients' skills and confidence in managing their health problems, including regular assessment of progress and problems, goal setting, and problem-solving support" [17]. The core components of the interventions were provider education, provider feedback, provider reminders, patient education, patient reminders, and patient financial incentives with elements that included standardized training, multidisciplinary team, peer contact, keeping logs, goal setting skills, problem solving skills, and seeking support.

\section{Outcomes}

Primary outcomes included clinical indicators such as blood pressure, eGFR, and $\mathrm{HbA}_{1 \mathrm{c}}$, and secondary outcomes included self-management activity, health service utilization, health-related quality of life (HRQOL), adherence to medications, and death.

\section{Study design}

Randomized controlled studies (including cluster randomized controlled trials) and systematic reviews of randomized controlled studies were considered. We included English-language peer-reviewed journal articles. We excluded articles reporting non-randomized studies, narrative reviews, letters, editorials, commentaries, 
Table 1 Selection criteria

\begin{tabular}{|c|c|c|}
\hline & Inclusion & Exclusion \\
\hline Participants & $\begin{array}{l}\text { Adult patients (above } 18 \text { years) with diabetes }{ }^{\mathrm{a}} \text { and } \\
\text { CKD in any health care setting }\end{array}$ & Participants without the diagnosis of diabetes and CKD \\
\hline Interventions & $\begin{array}{l}\text { Self-management models including at least one of } \\
\text { the following intervention components: } \\
\text { Provider education, provider feedback, provider } \\
\text { reminders, patient education, patient reminders, } \\
\text { and patient financial incentives }\end{array}$ & $\begin{array}{l}\text { No intervention or any intervention other than those } \\
\text { prespecified in the inclusion criteria }\end{array}$ \\
\hline Control & $\begin{array}{l}\text { Clearly defined usual or standard care. This may be } \\
\text { the chronic disease management programme that } \\
\text { is already in place before a new model of care is introduced }\end{array}$ & $\begin{array}{l}\text { Any intervention except those listed in the } \\
\text { inclusion criteria }\end{array}$ \\
\hline Outcomes & $\begin{array}{l}\text { Must include at least one of the following outcomes: } \\
\text { Primary: } \\
\text { 1. Clinical indicators (blood pressure, eGFR, and } \mathrm{HbA}_{1 \mathrm{c}} \text { ) } \\
\text { Secondary: } \\
\text { 1. Medication adherence } \\
\text { 2. Self-management activity } \\
\text { 3. Health service utilization including hospitalization } \\
\text { 4. Health-related quality of life } \\
\text { 5. Adverse events such as deaths }\end{array}$ & Lack of at least one relevant prespecified outcome \\
\hline Study design & $\begin{array}{l}\text { Randomized controlled trials and systematic reviews } \\
\text { of randomized controlled trials }\end{array}$ & Studies reporting non-randomized studies \\
\hline
\end{tabular}

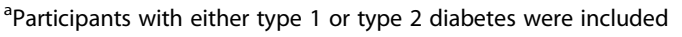

CKD chronic kidney disease which was defined as a sustained decrease in eGFR to levels less than $60 \mathrm{~mL} / \mathrm{min} / 1.73 \mathrm{~m}$ for a period of $3 \mathrm{months}$ or longer, eGFR estimated glomerular filtration rate, $H b A_{1 c}$ glycated hemoglobin

unpublished manuscripts, dissertations, government reports, books and book chapters, conference proceedings, meeting abstracts, lectures and addresses, and consensus development statements and guidelines.

\section{Literature search}

We conducted a comprehensive search of literature, which has been described in detail elsewhere [15]. In brief, we identified RCTs through Medline, Medline in-process and other non-indexed citations, EMBASE, CINAHL, and all evidence-based medicine (EBM) reviews. We also searched the bibliographies of relevant studies identified by the search strategy for identification of additional studies. The databases were searched from January 1, 1994, to December 19, 2017. A detailed description of search limits is provided elsewhere (Additional file 1: Table S1). To ensure reliability, two reviewers (EZ and CL) independently scanned the titles, abstract sections, and keywords of every article obtained by the search strategy. The two reviewers retrieved full texts of potentially relevant studies and screened them independently for inclusion. During the full-text review, if the two reviewers were in doubt about the inclusion of any particular study, the third reviewer $(\mathrm{MM})$ was involved. Investigators of all eligible studies were also contacted by email to request unpublished data relevant to the review.

\section{Data extraction and critical appraisal}

Two reviewers (EZ and CL) independently extracted data relevant to the PICO framework using a specially designed data abstraction form. Information was collected on general details (title, authors, reference/ source, country, year of publication, setting), participants (age, sex, inclusion/exclusion criteria, withdrawals/losses to follow-up, subgroups), results (point estimates and measures of variability, frequency counts for dichotomous variables, number of participants, intention-to-treat analysis), and validity results.

The methodological quality of each of the included studies was independently appraised by two reviewers (EZ and CL) using the Monash Centre for Health Research and Implementation (MCHRI) template [18] (Additional file 2: Table S2) and the quality of evidence using the Grading of Recommendations, Assessment, Development, and Evaluation (GRADE) approach [19]. Any disagreement was resolved by discussion with the third reviewer (MM) to reach a consensus. We contacted authors of included trials when clarification surrounding study conduct or missing data was required.

\section{Data synthesis and meta-analysis}

Analyses of data from included trials were performed with Review Manager (RevMan version 5.3.5, The Nordic Cochrane Centre, The Cochrane Collaboration, Copenhagen, Denmark). For meta-analysis, all outcomes were continuous and results are presented as mean differences (MDs) or standard mean difference (SMD) if different scales were used [12] with 95\% confidence interval (CI). A positive SMD value indicated the intervention group was superior to the control group on a positively oriented outcome measure. Data from eligible studies were pooled using the random effects model to 
account for heterogeneity [20]. Statistical heterogeneity was quantified using the inconsistency index- $I^{2}$ statistic with "low" heterogeneity set at $\leq 25 \%$, "moderate" $50 \%$, and "high" $\geq 75 \%$. To assess clinical heterogeneity, we performed a sensitivity analysis excluding a study of people with end-stage renal disease from the analysis. A subgroup analysis of pooled data based on the different self-management components was also carried out. Publication bias was not statistically assessed due to the small number of RCTs included. Statistical significance was set at $p<0.05$ for primary and secondary outcome measures. A descriptive analysis was performed to summarize data narratively for outcomes that had unexplained heterogeneity and missing data such as means and SDs and when there was a small number of studies reporting an outcome (less than 2 studies).

\section{Results}

\section{Literature search and study characteristics}

The results of the systematic search are shown in Fig. 1 . Two thousand and eighty references were identified by the search including 11 obtained from hand-searching of reference lists of seven systematic reviews [21-27] obtained from the search. After removal of duplicates and screening of titles and abstracts, 48 full-text articles were reviewed for further assessment. Following the full-text review, 40 articles were excluded based on reasons outlined in Fig. 1 and Additional file 3: Table S3. Eight studies [28-34] remained and were included in the systematic review. One of the studies (the SURE study) [30] had a duplicate publication [9], which reported on cost implication of the intervention. We treated the two publications as one study.

Characteristics of the eight included studies are presented in Table 2. Three studies were performed in the UK [28, 31, 34] and one each in Canada [29], China [30], USA [32], Netherlands [33], and Australia [35]. Four studies $[28,29,31,33]$ were conducted in a primary care setting, two in hospital-based outpatient clinics [34,35], one in hospital [30], and one in hemodialysis or peritoneal dialysis units [32]. Three studies [30, 33, 34] included patients with type 2 diabetes only; two studies [29, 32] specified

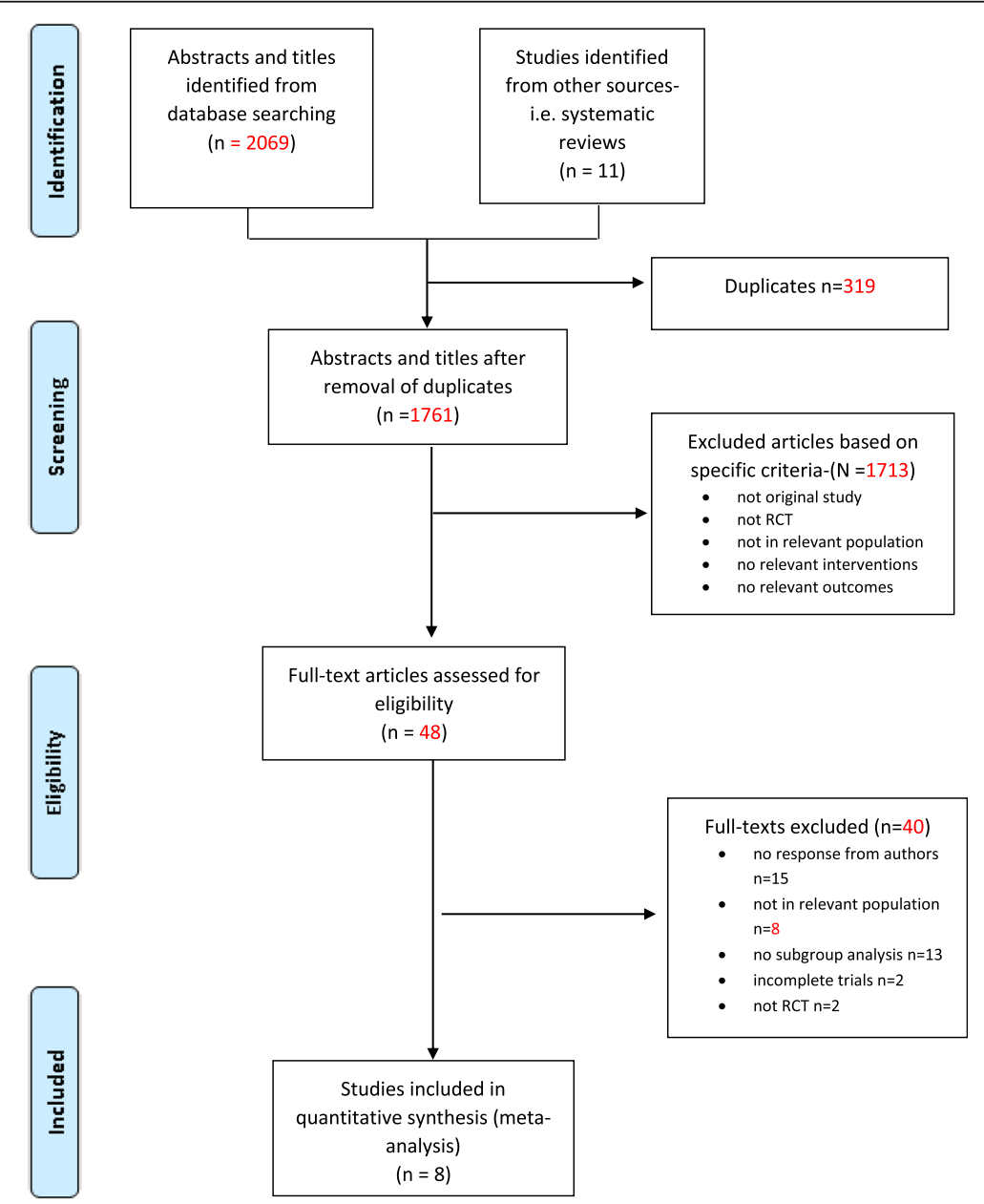

Fig. 1 PRISMA flow diagram showing how studies were screened [13] 


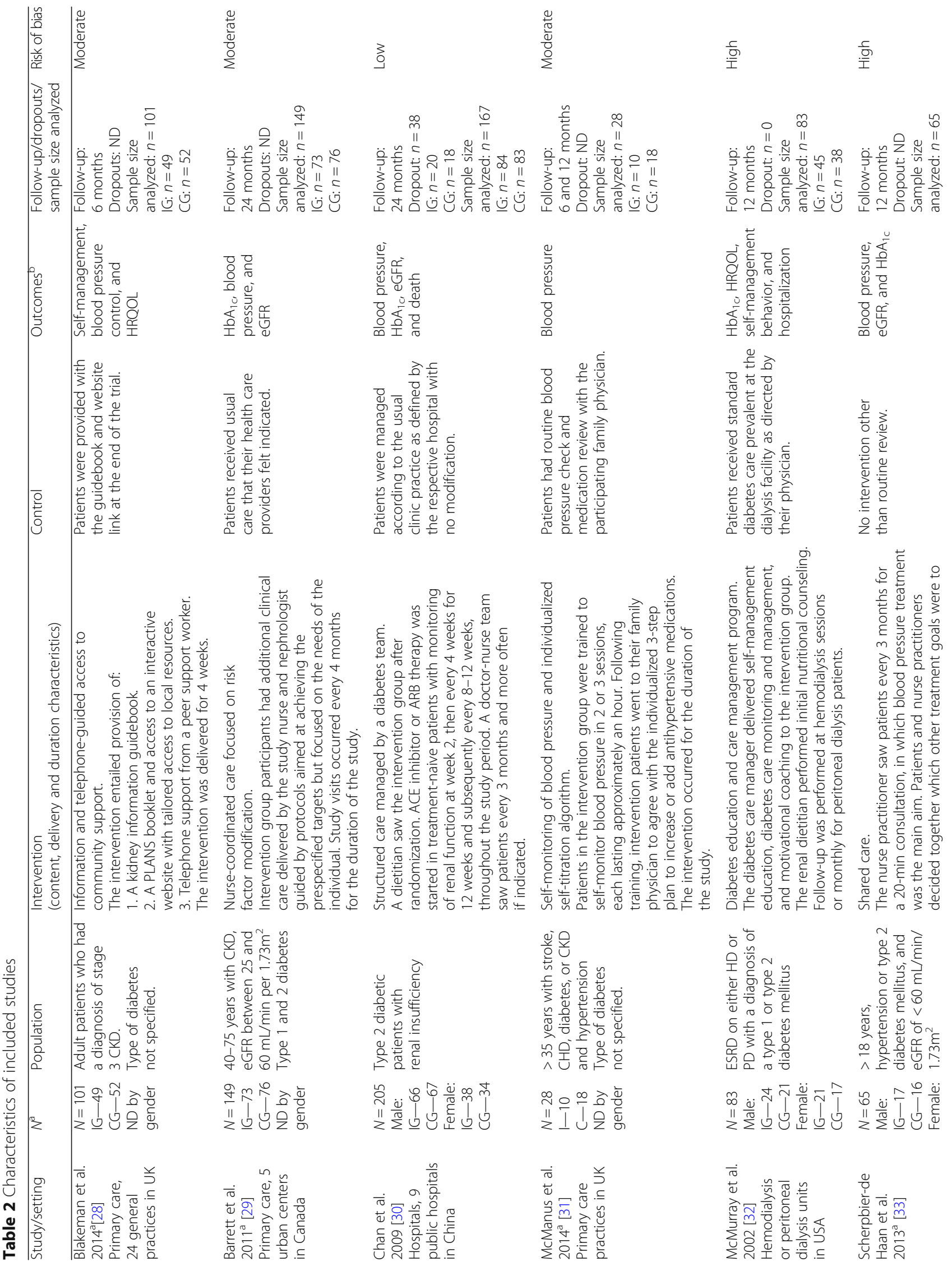




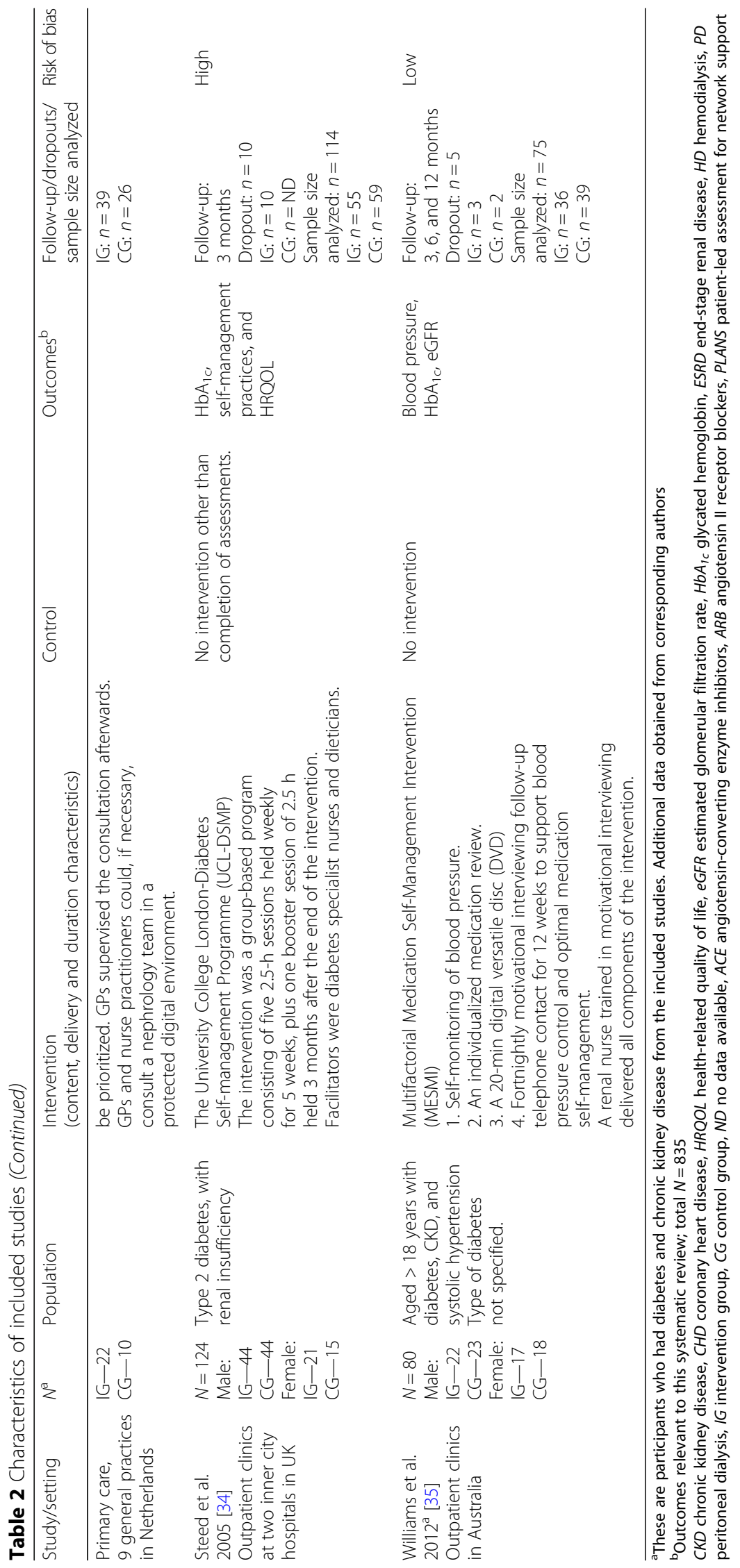


having patients with both type 1 and type 2 , and three studies [28, 31, 35] did not specify the type of diabetes. There was a substantial variation in the study sample sizes ( $n=28$ to 205), interventions, and follow-up period (3 to 24 months). Most of the studies were excluded due to inadequate data reported and no responses from authors $(N=15)$ and lack of evidence demonstrating that they included the correct population relevant to this review $(N=13)$ (Additional file 3: Table S3).

\section{Elements and components of self-management support interventions}

All the included studies had a theoretical underpinning for their self-management elements. Key elements of these interventions were derived from the Chronic Care Model [36], the Stanford Model [37], the Expert Patient Programme [38], and the Flinders Model [39] (Table 3). These elements include standardized training, multidisciplinary team, peer contact, keeping logs, goal setting and problem solving skills, and seeking support. There was a marked variation in the elements of interventions in terms of both content and delivery (Tables 2 and 3). Seven studies [28-34] described interventions underpinned by care coordination and a team based-approach with a focus on patient self-management and working collaboratively. The intervention components reported were patient education [28, 30-32, 34, 35], provider reminders [29, 30], and provider education [28, 33] (Fig. 2).

\section{Delivery characteristics}

The delivery characteristics for the interventions are shown in Table 2 . The study duration ranged from 3 to 24 months, with two studies having a duration of less than 12 months. The potential influence of follow-up duration on the estimates was explored by plotting the effect size against follow-up time, and there was no relationship between the two. Most of the studies had more than one delivery element. Five studies utilized face-to-face delivery
[30-32, 34, 35], three had the self-management component delivered by telephone [28, 30,35], and four used written information, websites, and protocols [28, 29, 31, $33]$ to guide the delivery of the interventions. All studies apart from one [35] had members of the multidisciplinary team facilitating the delivery of self-management support interventions. The members included nurses, dietitians, social workers, general practitioners, diabetologists, endocrine trainees, and nephrologists.

\section{Risk of bias in included studies}

Additional file 4: Figure S1 and Additional file 5: Figure S2 present an overview of the risk of bias for the included studies assessed against six risk-of-bias criteria which included selection (randomization and allocation), performance, detection, attrition, and reporting bias. Five studies [28-31, 35] reported random sequence generation, and four studies [28-30,35] demonstrated adequate allocation concealment. The majority of studies had high risk of performance bias $[28,29,31,32,34]$ and detection bias $[28,29,31$, $32,34]$. Only one study had a low risk of performance bias [33] and one study a low risk of detection bias [35]. Seven studies [28, 30-35] had a low risk of attrition bias, and all included studies had a low risk of reporting bias.

\section{Effects of interventions}

Table 4 provides the main comparison between groups, which had self-management support interventions, and controls. The study interventions were of varying intensity levels. Meta-analyses were only performed for systolic blood pressure, diastolic blood pressure, eGFR, $\mathrm{HbA}_{1 \mathrm{c}}$, diabetes self-management activity, and HRQOL.

\section{Primary outcomes \\ Systolic blood pressure}

Treatment effects for systolic blood pressure were reported by six studies [28-31, 33, 35] with mean systolic blood pressures ranging from 127 to $144 \mathrm{mmHg}$ for the

Table 3 Key elements to effective planned self-management support interventions

\begin{tabular}{|c|c|c|c|c|c|c|c|}
\hline Study & $\begin{array}{l}\text { Standardized } \\
\text { training }\end{array}$ & $\begin{array}{l}\text { Multidisciplinary } \\
\text { team }\end{array}$ & $\begin{array}{l}\text { Peer } \\
\text { contact }\end{array}$ & $\begin{array}{l}\text { Keeping } \\
\text { logs }\end{array}$ & $\begin{array}{l}\text { Goal setting } \\
\text { skills }\end{array}$ & $\begin{array}{l}\text { Problem solving } \\
\text { skills }\end{array}$ & $\begin{array}{l}\text { Seeking } \\
\text { support }\end{array}$ \\
\hline Blakeman et al. [28] & * & * & * & & $*$ & & $*$ \\
\hline Barrett et al. [29] & & * & & & & & \\
\hline Chan et al. [30] & $*$ & * & & & & & \\
\hline McManus et al. [31] & * & $*$ & & * & $*$ & * & $*$ \\
\hline McMurray et al. [32] & * & * & & & $*$ & * & \\
\hline Scherpbier-de Haan et al. [33] & $*$ & * & & & $*$ & & * \\
\hline Steed et al. [34] & * & $*$ & * & & $*$ & & \\
\hline Williams et al. [35] & $*$ & & & $*$ & $*$ & & $*$ \\
\hline
\end{tabular}

The studies utilized elements derived from the following self-management models: (a) the Chronic Care Model, (b) the Stanford Model, (c) the Expert Patient Programme, and (d) the Flinders Models

*means respective self-management element was used by the study 


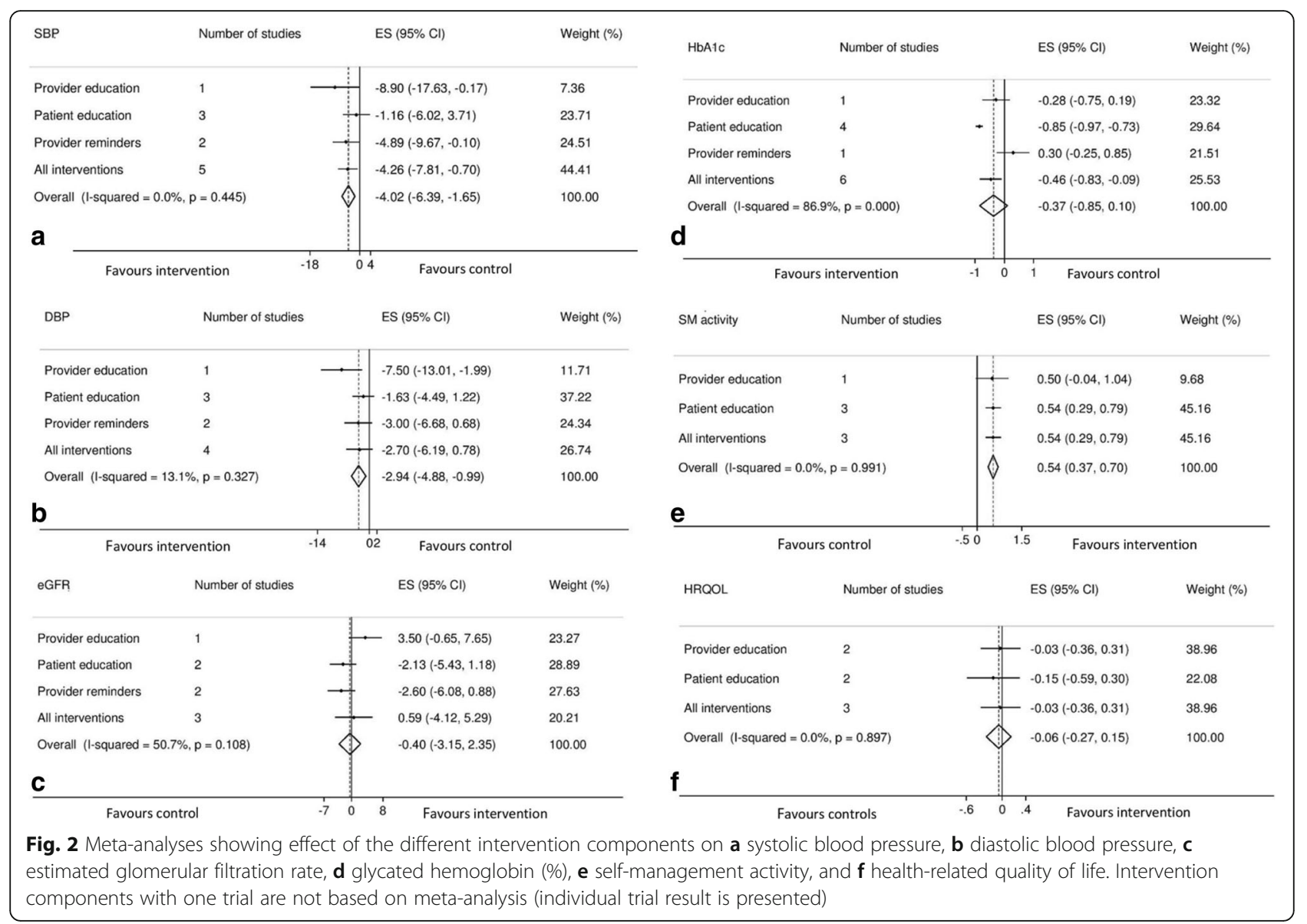

intervention groups and 134 to $146 \mathrm{mmHg}$ for the control groups. Two of the six studies [29, 33] utilizing structured care and shared care as interventions reported significant improvements in blood pressure in the intervention groups compared to the control groups. Barrett et al. [29] reported a mean difference (MD) of $-7.20 \mathrm{mmHg}$ (95\% CI -13.69 to $0.71, p<0.05$ ) between the intervention and control groups, while the study by Scherpbier-de Haan et al. [33] showed a MD of $-8.90 \mathrm{mmHg}$ (17.63 to -0.17 , $p<0.05$ ) (Fig. 3a). Data was pooled from five studies [29-31, 33, 35], which were deemed sufficiently homogenous to conduct a meta-analysis. The intervention group had a significantly lower systolic blood pressure than the control group [Fig. 3a; MD $-4.26 \mathrm{mmHg}(95 \%$ CI -7.81 to -0.70$) p=0.02]$.

\section{Diastolic blood pressure}

Four studies [30, 31, 33, 35] reported mean diastolic blood pressures ranging from 68 to $74 \mathrm{mmHg}$ for the intervention groups and 71 to $80 \mathrm{mmHg}$ for the control groups. Significantly lower diastolic blood pressures were reported in two studies by Chan et al. [30] and Scherpbier-de Haan et al. [33]: MDs in diastolic blood pressure of $-3 \mathrm{mmHg}(95 \% \mathrm{CI}-6.68$ to 0.68$)$ and $-7.5 \mathrm{mmHg}$
(95\% CI -13.01 to -1.99 ) respectively (Fig. 3b). Data from four studies was available for a meta-analysis. There was no significant difference in the diastolic blood pressure of the intervention and control groups [Fig. 3b; MD - 2.70 (95\% $\mathrm{CI}-6.19$ to 0.78$) p=0.13]$.

\section{Estimated glomerular filtration rate}

Estimated glomerular filtration rate was evaluated by four studies [29, 30, 33, 35]. Data from three [30, 33, 35] studies were available for a meta-analysis. The mean differences for eGFR among the three studies ranged from -2.6 to $3.5 \mathrm{~mL} / \mathrm{min} / 1.73 \mathrm{~m}^{2}$. There was no significant difference in the eGFR of the intervention and control groups [Fig. 3c; MD -0.59 (95\% CI -4.12 to 5.29) $p=0.81$. However, a moderate degree of heterogeneity was detected $\left(I^{2}=60 \%\right)$.

\section{Hemoglobin $A_{1 c}$}

Six studies $[29,30,32-35]$ reported mean $\mathrm{HbA}_{1 \mathrm{c}}$ levels ranging from 6.3 to $8.1 \%$ for the intervention groups and 7.1 to $8.5 \%$ for the control groups. Three studies [30, 32, 35] which included structured care managed by a diabetes team, diabetes education and care management program, and multifactorial medication self-management reported lower $\mathrm{HbA}_{1 \mathrm{c}}$ levels in the intervention groups (MDs 
Table 4 Summary of findings for the main comparison

Self-management compared with control for participants with diabetes and chronic kidney disease

Patient or population: patients with diabetes and chronic kidney disease

Settings: community, primary care, hospital outpatient

Intervention: self-management

Comparison: standard care

Outcomes Impact

Systolic blood pressure

Follow-up:

6 to 24 months

$[28-31,33,35]$

Diastolic blood pressure

Follow-up:

12 to 24 months

$[30,31,33,35]$

eGFR

Follow-up:

12 to 24 months

$[29,30,33,35]$

$\mathrm{HbA}_{1 \mathrm{c}}$

Follow-up:

3-24 months

[29, 30, 32-35]

Adherence to medications Follow-up: 12 months [35]

Self-management activity

Follow-up:

3-12 months

$[28,32,34]$

Health service utilization Follow-up: 6-24 months $[28,30,32]$

Health-related quality of life

Follow-up: 3-12 months [28, 32-34]

Death

Follow-up:

12 to 24 months

[30-32]

SBP MDs ranged from -8.90 to $3.60 \mathrm{mmHg}$. One study* [28] was excluded from the meta-analysis due to insufficient data.

DBP MDs -7.50 to $2.30 \mathrm{mmHg}$

Estimated GFR MDs ranged from -2.60 to

$3.50 \mathrm{~mL} / \mathrm{min} / 1.73 \mathrm{~m}^{2}$. One study* [29] was

excluded from the meta-analysis due to

insufficient data.

$\mathrm{HbA}_{1 \mathrm{c}} \mathrm{MDs}$ ranged from -0.90 to $0.30 \%$.

One study [35] identified no difference in medication adherence between the control and intervention groups using the Morisky scale.

The self-management SMDs for the three studies ranged from 0.31 to 0.99 .

Two studies $[28,30]$ showed no differences in hospitalization between the intervention and control groups and one study [32] reported that the study group had lower hospitalization rates.

Two studies $[28,33]$ showed no difference in quality of life between the intervention and control groups, and in the other two studies [32, 34], the intervention group showed a statistically significant improvement in the quality of life assessment.

The three studies showed no differences in mortality between the intervention and control groups.
$M D-2.70$

$(-6.19,0.78)$

Relative effect estimate $(95 \% \mathrm{Cl})$

$\mathrm{MD}-4.26$

$(-7.81,-0.71)$

MD 0.59

$(-4.12,5.29)$

MD $-0.46 \%$

$(-0.83,-0.09)$

Not estimable

$1(80)$

Moderate $^{4}$

SMD 0.56

$(0.15,0.97)$

Not estimable

3 (389)

Low $^{1}$

$\mathrm{SMD}-0.03$

$(-0.36,0.31)$

4 (373)

Moderate $^{1}$

Not estimable

3 (354)

Very low 1,6

High quality: further research is very unlikely to change our confidence in the estimate of effect. Moderate quality: further research is likely to have an important impact on our confidence in the estimate of effect and may change the estimate. Low quality: further research is very likely to have an important impact on our confidence in the estimate of effect and is likely to change the estimate. Very low quality: we are very uncertain about the estimate

$S B P$ systolic blood pressure, $M D$ s mean differences, $C l$ confidence interval, $D B P$ diastolic blood pressure, eGFR estimated glomerular filtration rate, $H b A_{1 c}$ glycated hemoglobin, SMD standard mean difference

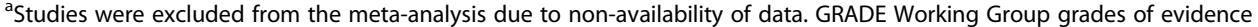

${ }^{1}$ The majority of the studies were not blinded to patients or outcome assessors and they did not report allocation concealment. The quality of evidence was downgraded by 2

${ }^{2}$ There was a considerable degree of inconsistency with several studies reporting effects in opposite directions. The quality of evidence was downgraded by 1

${ }^{3}$ One study reported on eGFR, but there was no data

${ }^{4}$ Relative estimate was not estimable. There were some discrepancies in responses as participants reported that they had no problem remembering to take their medications but at the same time they forgot to take their medications and vice versa. This study had allocation concealment and was blinded to investigators and outcome assessors. We did not downgrade based on limitations

${ }^{5}$ Heterogeneity was moderate $\left(l^{2}=63 \%\right)$. The $95 \%$ confidence intervals for some individual studies were narrower

${ }^{6}$ Death was reported by three studies (for the subgroup of patients with diabetes and chronic kidney disease), but the relative effect was not estimable 


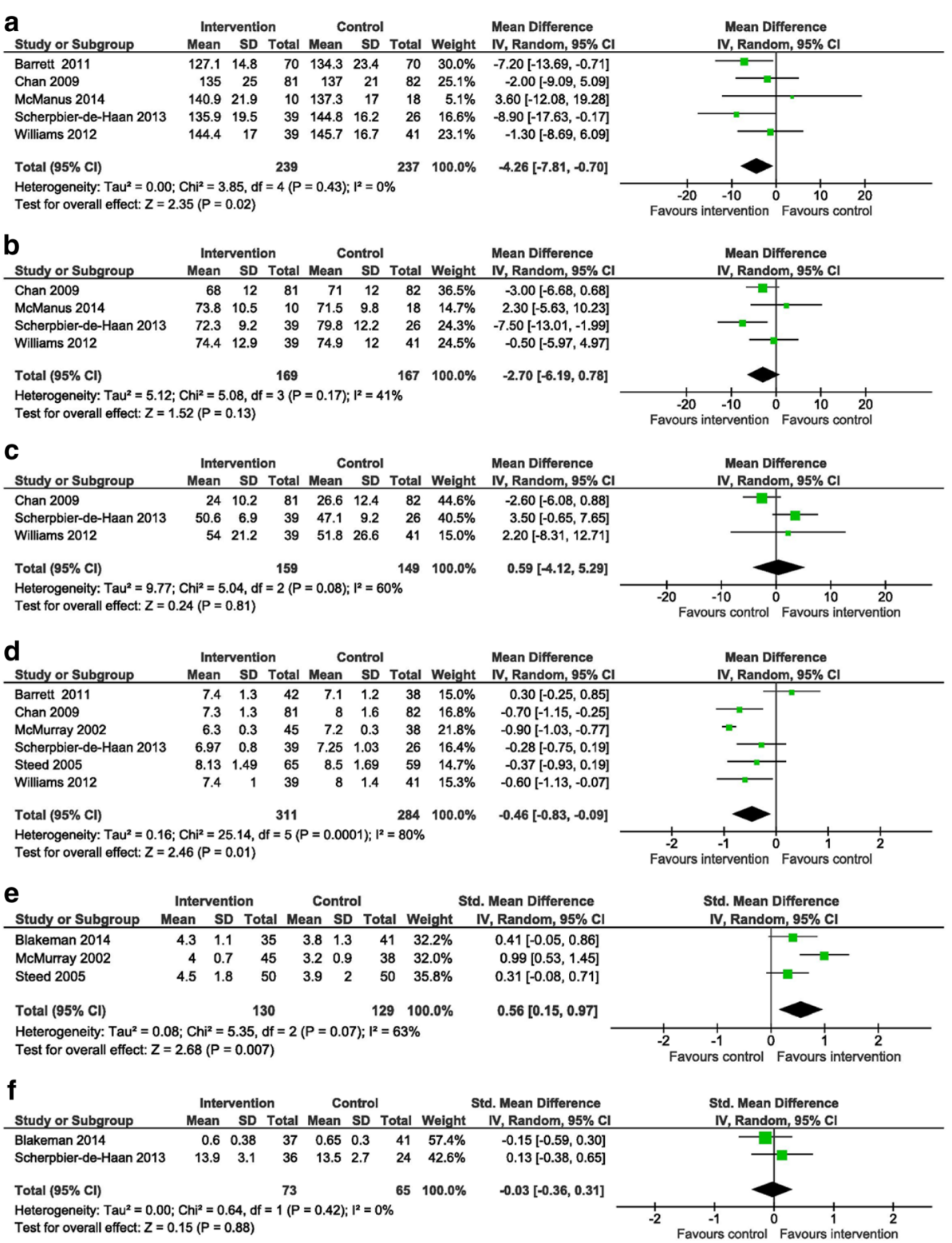

Fig. 3 Forest plots displaying the effectiveness of self-management support interventions in improving outcomes for patients with diabetes and chronic kidney disease: a systolic blood pressure, $\mathbf{b}$ diastolic blood pressure, $\mathbf{c}$ estimated glomerular filtration rate, $\mathbf{d}$ hemoglobin $\mathrm{A}_{1 \mathbf{c}}$ e self-management activity, and $\mathbf{f}$ health-related quality of life. The $x$-axis represents mean differences or standard mean differences. The $95 \%$ confidence intervals (Cl) for individual studies are represented by a horizontal line and by a diamond for pooled effect. SD standard deviation, IV inverse variance

ranging from -0.90 to $-0.60 \%$ ) than the control groups (Fig. 3d). In one study [29], which utilized the nurse-coordinated care intervention, there was a similar increase in the proportion of patients meeting $\mathrm{HbA}_{1 \mathrm{c}}$ targets in both the intervention and control groups. Data from the six studies were available for a meta-analysis. The intervention group had significantly lower $\mathrm{HbA}_{1 \mathrm{c}}$ levels than the control group [Fig. 3d; MD of $-0.5 \%$ (95\% CI -0.8 to -0.1$) p=0.01]$. However, a high degree of heterogeneity was detected $\left(I^{2}=80 \%, p=0.0001\right)$. A sensitivity analysis excluding the study with patients who had ESRD [32] confirmed that the intervention group had significantly lower $\mathrm{HbA}_{1 \mathrm{c}}$ levels than the control group [MD of $-0.3 \%(95 \% \mathrm{CI}-0.68$ to -0.01$) p=0.04]$.

\section{Secondary outcomes}

\section{Self-management activity}

Three studies [28, 32,34] assessed self-management activity and reported significant improvements in most self-management activities evaluated. Two studies utilized the Summary of Diabetes Self-Care Activity [28, 34] questionnaire, while one used the Diabetes Self-Care Knowledge 
questionnaire and Diabetes Self-Care Behaviour Inventory [32]. The SMD in self-care for the three studies ranged from 0.31 to 0.99 . Data from all three studies were included in a meta-analysis. There was a significant increase in self-care activities in the intervention groups compared to the control groups [Fig. 3e; SMD of 0.56 (95\% CI 0.15 to 0.97 ) $p=$ 0.007]. However, a moderate degree of heterogeneity was detected $\left(I^{2}=63 \%, p=0.07\right)$. A sensitivity analysis excluding the study with patients who had ESRD [32] showed significant improvements in most self-management activities evaluated [SMD of 0.35 (95\% CI 0.06 to 0.65) $p=0.02$ ].

\section{Health service utilization}

Three studies $[28,30,32]$ evaluated the effect of self-management support interventions on health service utilization. Chan et al. [30] reported similar rates of clinical events, hospitalization, and emergency room visits. Among the nine study sites, the structured care group reported lower event rates than the usual care group in five hospitals, higher event rates than the usual care group in two hospitals, and similar event rates in two hospitals. After a 2-year period, the structured care group were more likely to achieve three or more treatment goals $[61 \%(n=63)$ vs. $28 \%(n=28)]$ and those who attained three or more treatment goals $(n=91)$ had a $60 \%$ lower risk of the primary end point (death and/or renal end point creatinine $>500 \mu \mathrm{mol} / \mathrm{L}$ or dialysis) compared with those who did not attain three or more treatment goals $(n=114)[14$ vs. $34 ;$ RR $0.43(95 \%$ CI 0.21 to 0.86$)$ ]. Blakeman et al. [28] reported a mean (SD) service use of 7.6 (7.7) and 6.1 (3.6) for the intervention and control groups respectively $(p=0.27)$. McMurray et al. [32] reported a significant progression in diabetic-related peripheral vascular/neuropathic disease in the control group from a baseline score of 2.7 to a 12-month foot risk assessment score of 3.3 , whereas the study group did not show this progression $(p<0.02)$. The intervention group also had a statistically significant lower hospitalization rate for diabetes, peripheral vascular disease, infection, and amputation-related admissions $(p<0.05)$.

\section{Health-related quality of life}

Four studies examined health-related quality of life [28, 32-34]. Two studies [32, 34] which had missing summary data were not included in the meta-analysis. There was no significant difference in HRQOL scores between the intervention and control groups [Fig. 3f; SMD of -0.03 (95\% CI -0.36 to 0.31$) p=0.88]$. A low degree of heterogeneity was detected $\left(I^{2}=0 \%, p=\right.$ 0.42). All the four studies used different instruments for measuring HRQOL. Blakeman et al. [28] measured HRQOL with the EuroQol five dimensions questionnaire (EQ-5D) and reported no significant difference in mean (SD) EQ5D scores in the intervention and control groups respectively $(p=0.52)$. Steed et al. [34] showed differences in diabetes specific quality of life as measured by the Audit of Diabetes-Dependent Quality of Life (ADDQoL) questionnaire $(p<0.01)$. McMurray et al. [32] evaluated patient quality of life using a questionnaire adapted from the standardized Diabetes Form 2.1 and found that the intervention group had significant improvement in the quality-of-life assessment category of diabetes symptoms $(p<0.001)$. Scherpbier-de Haan et al. [33] reported no significant difference in mean (SD) WONCA scores in the intervention and control groups respectively $(p=0.40)$.

\section{Medication adherence}

Medication adherence was assessed in one study [35], which reported no difference in medication adherence between groups using pill counts. The mean adherence rate to the medications at the completion of the study was $66 \%$ in the control group and $58.4 \%$ in the intervention group $(p=0.16)$.

\section{Death}

Three studies [30-32] reported on death. Chan et al. [30] reported eight deaths in the structured care group $(N=104)$ and 11 in the usual care group $(N=101)$. In a study by McManus et al. [31], one patient died in each group and neither death was study-related. McMurray et al. reported no difference in mortality between the control and intervention groups.

\section{Discussion}

In this systematic review of eight studies among 835 patients with comorbid diabetes and CKD, there was moderate-quality evidence that self-management support interventions significantly improved self-management activity compared to usual care and low-quality evidence that these interventions significantly improved $\mathrm{HbA}_{1 \mathrm{c}}$ and systolic blood pressure but not diastolic blood pressure, eGFR, and HRQOL. The self-management components that were effective across these outcomes included provider reminders, patient education, and goal setting provided in multidisciplinary settings. In addition, treatment effects could not be quantitatively estimated for medication adherence, health service utilization, and death due to marked heterogeneity and insufficient data.

Our findings suggest that provider reminders, patient education, and goal setting may be associated with improved systolic blood pressure, $\mathrm{HbA}_{1 \mathrm{c}}$, and self-management activity. This is consistent with results from other studies among patients with hypertension [40] and type 2 diabetes mellitus [41]. Goal setting, reported in three studies [28, 32, 34], appeared to be an important self-management element to enhance self-care. This supports evidence from a previous study among patients with diabetes [42], which has suggested that a goal setting intervention along with a diabetes 
self-management guide help patients set and achieve healthy behavioral goals.

Although we found statistically significant increases in self-management activity with the self-management support interventions studied, the clinical relevance of these effects must be considered. A SMD of 0.5 has previously been reported as likely to represent a meaningful change or a minimal important difference in patient-reported outcomes [43, 44]. Our pooled estimate of 0.56 SMD units (range 0.15 to 0.97 ) thus suggests that an appreciable number of patients with diabetes and CKD may benefit from the self-management support interventions studied.

The Chronic Care Model (CCM) [45] provides a useful framework which explains how the multidisciplinary setting drives behaviour change especially for patients with complex diseases who require multi-faceted approaches to care. The benefits of the CCM include improved clinical outcomes [46-48], patient empowerment, and education [49]. Components of self-management support have been shown to be particularly effective when delivered by a multidisciplinary team for patients with CKD [50]. The reasons for this are that multidisciplinary members bring self-management expertise and they provide opportunities for further self-management support. In support of this, the KDIGO guidelines suggest that people with progressive CKD should be managed in a multidisciplinary care setting [51]. In this review, we cannot fully ascertain whether multidisciplinary settings led to the effectiveness of self-management support interventions since all included studies consisted of multidisciplinary teams.

These findings need to be considered in light of the very low to moderate quality of evidence examined. Reasons include potential biases in the methodological conduct of studies (including challenges in blinding investigators, participants, and outcome assessors in behavioral intervention studies [52]) and the small numbers of studies per outcome which limited interpretation of efficacy for the specific self-management support interventions investigated. There was marked heterogeneity especially for studies that reported on eGFR, $\mathrm{HbA}_{1 \mathrm{c}}$, and self-management activity. The reasons for this could be (1) the size of the included studies (small studies have been shown to be more heterogeneous than larger studies [53]) and (2) the variability related to the quality of the studies, characteristics of enrolled participants, and administered interventions. Our results could have also been biased by the exclusion of 28 studies due to non-response from corresponding authors and failure to specify subgroup analysis. Additionally, some studies compared interventions with usual care, which included key intervention components such as patient education, and specialist consult that could not be withheld due to ethical concerns $[29,30]$. Consequently, these biases may have weakened the effects of self-management support interventions on outcomes.
The review has a number of strengths. Firstly, to our knowledge, this is the first comprehensive review of evidence on self-management support interventions for patients with both diabetes and CKD. Secondly, this review is underpinned by the use of reliable tools, a peer-reviewed and published protocol, and rigorous methods that included efforts to retrieve additional methods, information, and data from study authors to ensure that accurate data were included and synthesized.

The review had a number of limitations. We excluded studies published in languages other than English. Another limitation was the assumption that self-management support interventions were standardized when practically many aspects of self-management, particularly those delivered outside the health care setting, are not. Therefore, we relied on subjective judgment to include or exclude studies when self-management support interventions were not explicitly stated. There was also considerable threat to internal validity due to the low quality of evidence from included studies stemming from difficulties in blinding of behavioral interventions [52]. Lastly, the interpretation of results from this review should take into consideration marked variation in self-management support interventions and outcome measures in the included studies.

Findings from this review have several implications to research and practice. First, a gap of research focusing on diabetes self-management support interventions and outcomes for patients with comorbid diabetes and CKD has been highlighted. Future research should therefore focus on studies designed primarily for people with both diabetes and CKD, and when a study among people with other chronic diseases includes this sub-population, a consistent approach to the conduct and reporting of secondary analysis should be rigorously followed. Second, there should be standardization of outcome measures such as HRQOL to reduce between-study heterogeneity and more studies should measure hard clinical end points and patient-reported outcomes like medication adherence. Additionally, we have shown that self-management support interventions may improve outcomes for people with comorbid diabetes and CKD, but the effect of these interventions beyond 24 months and the intensity of the interventions required still need to be explored. Well-designed longitudinal studies that compare the components of multifaceted interventions are required to understand which components are essential for producing beneficial effects. Such studies may also gather data essential for the development of a complex RCT that can test self-management as an intervention.

\section{Conclusion}

Self-management support interventions may improve self-care activities, systolic blood pressure, and $\mathrm{HbA}_{1 \mathrm{c}}$ in patients with comorbid diabetes and CKD. This evidence is 
based on low to moderate quality studies with relatively few study participants. It was not possible to determine which self-management support components and elements were more effective, but interventions that utilized provider reminders, patient education, and goal setting provided in multidisciplinary settings were associated with improved outcomes. More evidence from high-quality studies is required to support future self-management programs.

\section{Additional files}

Additional file 1: Table S1. Ovid MEDLINE search strategy conducted on 19 December 2017. (DOCX 17 kb)

Additional file 2: Table S2. Template for critical appraisal of a randomized controlled trial. (DOCX $20 \mathrm{~kb}$ )

Additional file 3: Table S3. Characteristics of excluded studies (ordered alphabetically). (DOCX $12 \mathrm{~kb}$ )

Additional file 4: Figure S1. Risk of bias: review authors' judgements about each risk of bias item presented as percentages across all included studies. (PNG 205 kb)

Additional file 5: Figure S2. Methodological quality summary: review authors' judgements about each methodological quality item for each included study. (PNG 583 kb)

\section{Abbreviations}

ADDQoL: Audit of Diabetes-Dependent Quality of Life; CCM: Chronic Care Model; Cl: Confidence intervals; CINAHL: Cumulative Index to Nursing and Allied Health Literature; CKD: Chronic kidney disease; EBM: Evidence-based medicine; eGFR: Estimated glomerular filtration rate; EMBASE: Excerpta Medica database; EQ5D: European Quality of Life-5 Dimensions questionnaire; ESRD: End-stage renal disease; GRADE: Grading of Recommendations, Assessment, Development, and Evaluation; $\mathrm{HbA}_{1 \mathrm{c}}$ : Glycated hemoglobin; HRQOL: Health-related quality of life; MCHRI: Monash Centre for Health Research and Implementation; MD: Mean difference; PICO: Population, Intervention, Comparison, and Outcome; PRISMA: Preferred Reporting Items for Systematic Reviews and Meta-Analyses; RCTs: Randomized controlled trials; SDSCA: Summary of Diabetes Self-Care Activity questionnaire: SMD: Standard mean difference; SURE study: Structured versus usual care on renal endpoint in type 2 diabetes; WONCA scores: World Organization of National Colleges, Academies, and Academic Associations of General Practitioners/Family Physicians

\section{Acknowledgements}

We thank Blakeman et al., Barret et al., McManus et al., Scherpbier-de Haan et al., and Williams et al. for providing additional data required for this review.

\section{Availability of data and materials}

All materials relevant to this review including results of the search, included and excluded studies, the PRISMA flow diagram, and risk of bias and assessment of methodological quality are included in the article and additional information files. Data abstraction forms are available from the corresponding author on request.

\section{Authors' contributions}

$E Z, C L, S R, M M, P G K, H J T$, and SZ contributed to the research idea and study design. EZ, CL, MM, SR, and SZ contributed to the data acquisition. EZ, CL, SR, and SZ contributed to the data analysis/interpretation. EZ, CL, SR, and SZ contributed to the statistical analysis. SZ, PGK, and HJT contributed to the supervision or mentorship. Each author contributed significant intellectual content during the drafting of the manuscript and revisions and accepts accountability for the overall work. All authors read and approved the final manuscript.

\section{Ethics approval and consent to participate} Not applicable.

\section{Competing interests}

The authors declare that they have no competing interests.

\section{Publisher's Note}

Springer Nature remains neutral with regard to jurisdictional claims in published maps and institutional affiliations.

\section{Author details}

${ }^{1}$ Monash Centre for Health Research and Implementation, School of Public Health and Preventive Medicine, Monash University, 43-51 Kanooka Grove, Clayton, Melbourne, Victoria, Australia. ${ }^{2}$ Department of Nephrology, Monash Health, Melbourne, Victoria, Australia. ${ }^{3}$ Diabetes and Vascular Medicine Unit, Monash Health, Melbourne, Victoria, Australia. ${ }^{4}$ Department of Medicine, School of Clinical Sciences at Monash Health, Monash University, Melbourne, Victoria, Australia. ${ }^{5}$ The George Institute for Global Health, University of Sydney, Sydney, New South Wales, Australia.

Received: 20 November 2017 Accepted: 24 May 2018

Published online: 13 June 2018

\section{References}

1. Guariguata L, Whiting DR, Hambleton I, Beagley J, Linnenkamp U, Shaw JE. Global estimates of diabetes prevalence for 2013 and projections for 2035. Diabetes Res Clin Pract. 2014:103(2):137-49.

2. Ogurtsova K, da Rocha Fernandes JD, Huang Y, et al. IDF diabetes atlas: global estimates for the prevalence of diabetes for 2015 and 2040. Diabetes Res Clin Pract. 2017;128:40-50.

3. Chowdhury TA, Shaho S, Moolla A. Complications of diabetes: progress, but significant challenges ahead. Ann Transl Med. 2014;2(12):120.

4. Mills KT, Xu Y, Zhang W, et al. A systematic analysis of worldwide population-based data on the global burden of chronic kidney disease in 2010. Kidney Int. 2015;88(5):950-7.

5. Schernthaner G. Kidney disease in diabetology. Nephrol Dialysis Transplantation. 2007;22(3):703-7.

6. Tuttle KR, Bakris GL, Bilous RW, et al. Diabetic kidney disease: a report from an ADA consensus conference. Am J Kid Dis. 2014;64(4):510-33.

7. Palsson R, Patel UD. Cardiovascular complications of diabetic kidney disease Adv Chronic Kidney Dis. 2014;21(3):273-80.

8. Zimbudzi E, Lo C, Ranasinha $\mathrm{S}$, et al. Predictors of health-related quality of life in patients with co-morbid diabetes and chronic kidney disease. PLoS One. 2016;11(12):e0168491.

9. Ko GT, Yeung CY, Leung WY, et al. Cost implication of team-based structured versus usual care for type 2 diabetic patients with chronic renal disease. Hong Kong Med J. 2011;17(6):9-12

10. Welch JL, Johnson M, Zimmerman L, Russell CL, Perkins SM, Decker BS. Selfmanagement interventions in stages 1 to 4 chronic kidney disease: an integrative review. West J Nurs Res. 2015;37(5):652-78.

11. Griva K, Mooppil N, Khoo E, Lee WW, Kang AWC, Newman SP. Improving outcomes in patients with coexisting multimorbid conditions - the development and evaluation of the combined diabetes and renal control trial (C-DIRECT): study protocol. BMJ Open. 2015;5(2):e007253.

12. Higgins JPT, Green S. Cochrane Handbook for Systematic Reviews of Interventions Version 5.1.0 [updated March 2011]. The Cochrane Collaboration; 2011. Available from www.handbook.cochrane.org.

13. Moher DL, Tetzlaff A, Altman J, G D. Preferred reporting items for systematic reviews and meta-analyses: the PRISMA statement. PLoS Med. 2009;6(7): e1000097.

14. Zimbudzi E LC, Misso M, Ranhasinha S, Zoungas S. Effectiveness of management models for facilitating self-management and patient outcomes in people with diabetes and chronic kidney disease PROSPERO 2015:CRD42015017316; [Available from: https://www.crd.york.ac.uk/ prospero/display_record.php?RecordID=17316. Accessed 10 Mar 2017

15. Zimbudzi E, Lo C, Misso M, Ranasinha S, Zoungas S. Effectiveness of management models for facilitating self-management and patient outcomes in adults with diabetes and chronic kidney disease. Syst Rev. 2015:4:81.

16. The National Kidney Foundation (NKF). Kidney Disease Outcomes Quality Initiative (K/DOQI) clinical practice guidelines for chronic kidney disease: evaluation, classification, and stratification. Am J Kidney Dis. 2002;39(2-1):S1-266.

17. Adams K, Greiner AC, Corrigan JM. Report of a summit. The 1st annual crossing the quality chasm summit-a focus on communities. Washington, DC: National Academies Press; 2004 
18. Monash Centre for Health Research and Implementation (MCHRI). Evidence Synthesis Program templates for critical appraisal and risk of bias (adapted from Critical Appraisal Templates, Centre for Clinical Effectiveness, Southern Health, Melbourne, 2010). Melbourne: MCHRI-Monash University and Monash Health; 2013.

19. Guyatt GH, Oxman AD, Schünemann HJ, Tugwell P, Knottnerus A. GRADE guidelines: a new series of articles in the journal of clinical epidemiology. J Clin Epidemiol. 2011;64(4):380-2.

20. Viechtbauer W. Accounting for heterogeneity via random-effects models and moderator analyses in meta-analysis. Z Psychol. 2007;215(2):104-21.

21. Strand H, Parker D. Effects of multidisciplinary models of care for adult predialysis patients with chronic kidney disease: a systematic review. Int J Evid Based Healthc. 2012;10(1):53-9.

22. McDonald HP, Garg AX, Haynes RB. Interventions to enhance patient adherence to medication prescriptions: scientific review. JAMA. 2002; 288(22):2868-79.

23. Santschi V, Chiolero A, Burnand B, Colosimo AL, Paradis G. Impact of pharmacist care in the management of cardiovascular disease risk factors: a systematic review and meta-analysis of randomized trials. Arch Intern Med. 2011;171(16):1441-53.

24. Roshanov PS, Misra S, Gerstein HC, et al. Computerized clinical decision support systems for chronic disease management: a decision-makerresearcher partnership systematic review. Implement Sci. 2011;6:92.

25. Bowles $\mathrm{KH}$, Baugh AC. Applying research evidence to optimize telehomecare. J Cardiovasc Nurs. 2007:22(1):5-15.

26. Sintchenko V, Magrabi F, Tipper S. Are we measuring the right end-points? Variables that affect the impact of computerised decision support on patient outcomes: a systematic review. Med Inform Internet Med. 2007;32(3):225-40.

27. Li T, Wu HM, Wang F, et al. Education programmes for people with diabetic kidney disease. Cochrane Database Syst Rev. 2011;(6):Cd007374.

28. Blakeman T, Blickem C, Kennedy A, et al. Effect of information and telephone-guided access to community support for people with chronic kidney disease: randomised controlled trial. PLoS One. 2014;9(10):e109135.

29. Barrett BJ, Garg AX, Goeree R, Levin A, Molzahn A, Rigatto C, Singer J, Soltys G, Soroka S, Ayers D, Parfrey PS. A nurse-coordinated model of care versus usual care for stage 3/4 chronic kidney disease in the community: a randomized controlled trial. Clin J Am Soc Nephrol. 2011;6(6):1241-7.

30. Chan JC, So WY, Yeung CY, et al. Effects of structured versus usual care on renal endpoint in type 2 diabetes: the SURE study: a randomized multicenter translational study. Diabetes Care. 2009:32(6):977-82.

31. McManus RJ, Mant J, Haque MS, et al. Effect of self-monitoring and medication self-titration on systolic blood pressure in hypertensive patients at high risk of cardiovascular disease: the TASMIN-SR randomized clinical trial. JAMA. 2014;312(8):799-808.

32. McMurray SD, Johnson G, Davis S, McDougall K. Diabetes education and care management significantly improve patient outcomes in the dialysis unit. Am J Kidney Dis. 2002:40(3):566-75.

33. Scherpbier-de Haan ND, Vervoort GM, van Weel C, et al. Effect of shared care on blood pressure in patients with chronic kidney disease: a cluster randomised controlled trial. Br J Gen Pract. 2013;63(617):e798-806.

34. Steed L, Lankester J, Barnard M, Earle K, Hurel S, Newman S. Evaluation of the UCL diabetes self-management programme (UCL-DSMP): a randomized controlled trial. J Health Psychol. 2005;10(2):261-76.

35. Williams A, Manias E, Walker R, Gorelik A. A multifactorial intervention to improve blood pressure control in co-existing diabetes and kidney disease: a feasibility randomized controlled trial. J Adv Nurs. 2012;68(11):2515-25.

36. Wagner EH, Austin BT, Von Korff M. Organizing care for patients with chronic illness. Milbank Q. 1996;74(4):511-44.

37. Lorig KR, Sobel DS, Stewart AL, et al. Evidence suggesting that a chronic disease self-management program can improve health status while reducing hospitalization: a randomized trial. Med Care. 1999;37(1):5-14.

38. Rogers A, Kennedy A, Bower P, et al. The United Kingdom Expert Patients Programme: results and implications from a national evaluation. Med J Aust. 2008;189(10):S21-4.

39. Battersby M, Harvey P, Mills PD, et al. SA HealthPlus: a controlled trial of a statewide application of a generic model of chronic illness care. Milbank Q. 2007;85(1):37-67

40. Bosworth HB, Olsen MK, Grubber JM, et al. Two self-management interventions to improve hypertension control: a randomized trial. Ann Intern Med. 2009:151(10):687-95.
41. Chrvala CA, Sherr D, Lipman RD. Diabetes self-management education for adults with type 2 diabetes mellitus: a systematic review of the effect on glycemic control. Patient Educ Couns. 2016;99(6):926-43.

42. DeWalt DA, Davis TC, Wallace AS, et al. Goal setting in diabetes self-management: taking the baby steps to success. Patient Educ Couns. 2009;77(2):218-23.

43. Norman GR, Sloan JA, Wyrwich KW. Interpretation of changes in healthrelated quality of life: the remarkable universality of half a standard deviation. Med Care. 2003;41(5):582-92.

44. Cuijpers P, Turner EH, Koole SL, van Dijke A, Smit F. What is the threshold for a clinically relevant effect? The case of major depressive disorders. Depress Anxiety. 2014;31(5):374-8.

45. Wagner $\mathrm{EH}$. Chronic disease management: what will it take to improve care for chronic illness? Eff Clin Pract. 1998;1(1):2-4.

46. MacLean CD, Littenberg B, Gagnon M, Reardon M, Turner PD, Jordan C. The Vermont Diabetes Information System (VDIS): study design and subject recruitment for a cluster randomized trial of a decision support system in a regional sample of primary care practices. Clinical trials (London, England). 2004;1(6):532-44.

47. Piatt GA, Orchard TJ, Emerson S, et al. Translating the chronic care model into the community: results from a randomized controlled trial of a multifaceted diabetes care intervention. Diabetes Care. 2006;29(4):811-7.

48. Smith SA, Shah ND, Bryant SC, et al. Chronic care model and shared care in diabetes: randomized trial of an electronic decision support system. Mayo Clin Proc. 2008;83(7):747-57.

49. Siminerio LM, Piatt G, Zgibor JC. Implementing the chronic care model for improvements in diabetes care and education in a rural primary care practice. Diabetes Educ. 2005;31(2):225-34.

50. Chen YR, Yang Y, Wang SC, et al. Effectiveness of multidisciplinary care for chronic kidney disease in Taiwan: a 3-year prospective cohort study. Nephrol Dial Transplant. 2013;28(3):671-82.

51. Kidney Disease Improving Global Outcomes (KDIGO CKD) Work Group. "Chapter 5: referral to specialists and models of care" in KDIGO 2012 Clinical Practice Guideline for the Evaluation and Management of Chronic Kidney Disease Kidney Int Suppl. 2013;3(1):112-119.

52. Friedberg JP, Lipsitz SR, Natarajan S. Challenges and recommendations for blinding in behavioral interventions illustrated using a case study of a behavioral intervention to lower blood pressure. Patient Educ Couns. 2010;78(1):5-11.

53. IntHout J, loannidis JP, Borm GF, Goeman JJ. Small studies are more heterogeneous than large ones: a meta-meta-analysis. J Clin Epidemiol. 2015:68(8):860-9.

\section{Ready to submit your research? Choose BMC and benefit from:}

- fast, convenient online submission

- thorough peer review by experienced researchers in your field

- rapid publication on acceptance

- support for research data, including large and complex data types

- gold Open Access which fosters wider collaboration and increased citations

- maximum visibility for your research: over $100 \mathrm{M}$ website views per year

At BMC, research is always in progress.

Learn more biomedcentral.com/submissions 